\title{
dspace.vutbr.cz
}

\section{Fully-Differential Universal Frequency Filter with Dual-Parameter Control of the Pole Frequency and Quality Factor}

\author{
LANGHAMMER, L.; ŠOTNER, R.; DVOŘÁK, J.; JEŘÁBEK, J.; ZAPLETAL, M.
}

Proceedings of the 2018 IEEE International Symposium on Circuits and Systems (ISCAS)

pp. 1-5

elSBN: 978-1-5386-4881-0

DOI: http://dx.doi.org/10.1109/ISCAS.2018.8351005

Accepted manuscript

(C)2018 IEEE. Personal use of this material is permitted. Permission from IEEE must be obtained for all other uses, in any current or future media, including reprinting/republishing this material for advertising or promotional purposes, creating new collective works, for resale or redistribution to servers or lists, or reuse of any copyrighted component of this work in other works. Lukas Langhammer, Roman Sotner, Jan Dvorak, Jan Jerabek, Miroslav Zapletal, " FullyDifferential Universal Frequency Filter with Dual-Parameter Control of the Pole Frequency and Quality Factor", Proceedings of the 2018 IEEE International Symposium on Circuits and Systems (ISCAS), pp. 1-5, 2017. DOI: 10.1109/ISCAS.2018.8351005. Final version is available at https://ieeexplore.ieee.org/document/8351005/ 


\section{Fully-Differential Universal Frequency Filter with Dual-Parameter Control of the Pole Frequency and Quality Factor}

\author{
Lukas Langhammer, Roman Sotner \\ Department of Radio Electronics, Faculty of Electrical \\ Engineering and Communication, Brno University of \\ Technology \\ Technicka 12, 61600, Brno, Czech Republic \\ langhammer@phd.feec.vutbr.cz
}

\author{
Jan Dvorak, Jan Jerabek, Miroslav Zapletal \\ Department of Telecommunications, Faculty of Electrical \\ Engineering and Communication, Brno University of \\ Technology \\ Technicka 12, 61600, Brno, Czech Republic
}

\begin{abstract}
The paper presents a proposal of modified $2^{\text {nd }}$ order fully-differential universal frequency filter. Both the pole frequency and quality factor (controlled without disturbing each other) of the filter can be controlled by two independent parameters (i.e. dual-parameter control) in order to increase the range of their control. It is the main improvement in comparison to recent state-of-the-art. The function of the proposed filter has been verified by PSpice simulations and also by experimental measurements.
\end{abstract}

Keywords-adjustable current amplifier; dual-parameter control; fully-differential; frequency filter; operational transconductance amplifier

\section{INTRODUCTION}

Although the digital signal processing is in the forefront nowadays, analogue frequency filters are still a necessity for wide range of electrical circuits. Thus, frequency filters which possess ability to adjust some of their parameters such as its pole frequency, quality factor, bandwidth, or gain in the passband area are beneficial. Moreover, the controllability provided in wider range is highly advantageous.

Controllability of the filter can be achieved a) electronically by adjusting one or more parameters of active element(s) [1-4], b) partly electronically by adjusting one parameter of used active element(s) and value of some passive part(s) [5], [6], c) by variation of values of passive elements [7-9] only. Transconductance $g_{\mathrm{m}}[1-3],[5],[6]$ and current gain $B$ [1], [4], [6] are typical used adjustable parameters of active elements. These parameters use to be controlled by DC voltage or current. Other possible parameter used for adjustability is intrinsic impedance $R_{\mathrm{X}}$ of used active element controlled by DC current [2-4] or also transresistance $R_{\mathrm{T}}$ [5]. In case of control by adjusting values of passive elements, adjusting values of resistors (conductances), capacitors [7-9] is only possible way. Table I provides a comparison of significant prior dual-parameter control filter designs.

The proposed filter provides the dual-parameter control of its pole frequency and quality factor and it has been designed in its fully-differential (F-D) form due to the advantages of F-D structures when compared to the single-ended (S-E) circuits such as greater dynamic range of the processed signals, better power supply rejection ratio, lower harmonic distortion and greater attenuation of common-mode signal [10].

\section{PROPOSAL DESCRIPTION}

The proposed filter is a modification of previously proposed filter from [5] in order to provide a dual-parameter control ability to electronically tune the pole frequency and quality factor of the filter without disturbing each other. The presented filtering structure is designed using multi-output

TABLE I. COMPARISON OF THE THEORETICAL, SIMULATED AND MEASURED POLE FREQUENCIES

\begin{tabular}{|c|c|c|c|c|c|c|c|}
\hline Ref No. & [1] & {$[2]$} & [3] & [4] & [5] & [6] & Fig. 4 \\
\hline Dual-param. control of $f_{0} / Q$ & Yes/No & Yes/No & Yes/Yes & Yes/No & $\mathrm{No} / \mathrm{Yes}$ & Yes/Yes & Yes/Yes \\
\hline Type of control & $g_{\mathrm{m}} / B$ & $R_{\mathrm{X}} / g_{\mathrm{m}}$ & $R_{\mathrm{X}} / g_{\mathrm{m}}$ & $R_{\mathrm{X}} / B$ & $g_{\mathrm{m}} / R_{\mathrm{T}}$ & $g_{\mathrm{m}} / B$ & $g_{\mathrm{m}} / R_{\mathrm{T}}$ \\
\hline Results & measured & simulated & simulated & simulated & measured & simulated & measured \\
\hline Tested range of $f_{0}[\mathrm{kHz}]$ & $38-2244$ & $1750-7520$ & $119-991$ & $43-4190$ & - & $800-2500$ & $36-2897$ \\
\hline$f_{0} \min -f_{0} \max$ ratio $[-]$ & 59.1 & 4.3 & 8.3 & 97.4 & - & 3.1 & 80.5 \\
\hline Tested range of $Q[-]$ & - & - & $1-16$ & - & $0.47-2.83$ & $0.6-1.9$ & $0.44-8.3$ \\
\hline$Q \min -Q \max$ ratio [-] & - & - & 16 & - & 6.0 & 3.2 & 18.9 \\
\hline
\end{tabular}

Research described in this paper was financed by the National Sustainability Program under grant LO1401. For the research, infrastructure of the SIX Center was used. 
transconductance amplifiers (MOTAs) [11], adjustable current amplifiers (ACAs) [12] and transresistance amplifier (OTRA) [13]. The MOTA element consists of two voltage input and four current output terminals and its schematic symbol is shown in Fig. 1 a). Relations between input and output terminals of the MOTA can be described as $I_{\text {OUT }+}=-I_{\text {OUT- }}=$ $g_{\mathrm{m}}\left(V_{\mathrm{IN}+}-V_{\mathrm{IN}-}\right)$, where $g_{\mathrm{m}}$ is the transconductance of this element. Figure $1 \mathrm{~b}$ ) shows a possible implementation of the MOTA using a universal current conveyor (UCC) [14], which has been developed in cooperation of Brno University of Technology and ON Semiconductor design center.

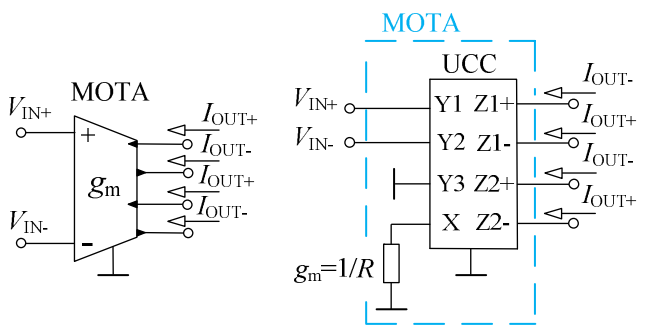

a)

b)
Fig. 1. Multi-Output Transconductance Amplifier: a) schematic symbol, b) possible implementation of the MOTA using the UCC

The ACA element consists of two current differential input and two or four current differential output terminals. Its schematic symbol and possible implementation using a UCC, universal voltage conveyor (UVC) [15] and EL2082 [16] is depicted in Fig 2 a), b), respectively. The relation between input and output terminals are given by $I_{\mathrm{OUT}+}=-I_{\mathrm{OUT}-}=B\left(I_{\mathrm{IN}+}-\right.$ $\left.I_{\mathrm{IN}-}\right)$, where $B$ is the current gain of this element.

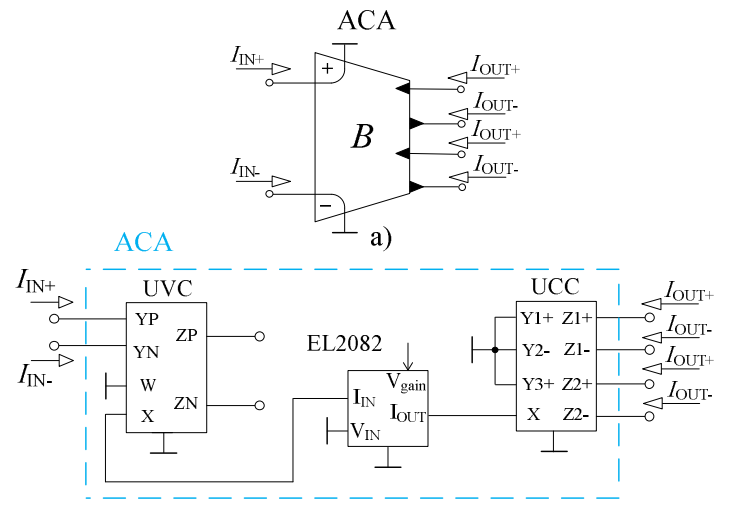

b)

Fig. 2. Adjustable Current Amplifier: a) schematic symbol, b) possible implementation of the ACA using the UCC, UVC and EL2082

The schematic symbol of the OTRA element together with its possible implementation using two UCC chips (each chip contains one UCC and one CCII) is illustrated in Fig. $3 \mathrm{a}$ ), b). This element consists of two current input and two voltage output terminals. The OTRA can be described by $v_{\mathrm{O}^{+}}=R_{\mathrm{T}}\left(I_{\mathrm{P}}-\right.$ $\left.I_{\mathrm{N}}\right), v_{\mathrm{O}-}=-R_{\mathrm{T}}\left(I_{\mathrm{P}}-I_{\mathrm{N}}\right)$, where $R_{\mathrm{T}}$ is the transresistance of this element.

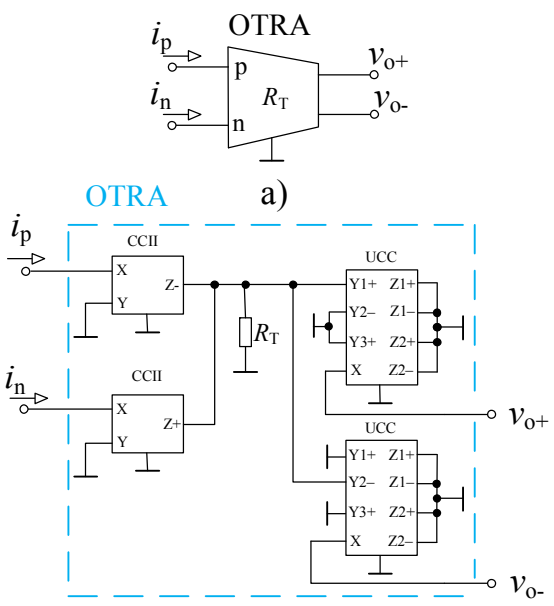

b)

Fig. 3. Operational Transresistance Amplifier: a) schematic symbol, b) possible implementation of the OTRA using two UCCs and two CCIIs

The proposed circuit structure of F-D filter is shown in Fig. 4. Since the floating capacitors are not suitable for implementation and integrability, floating capacitors in the filter structure have been replaced by grounded ones as can be seen in Fig. 4.

The denominator which is common for all obtainable transfer functions of the filter is given as:

$$
D(\boldsymbol{s})=\boldsymbol{s}^{2} C_{1} C_{2}+\boldsymbol{s} C_{2} g_{m 1} g_{m 3} B_{1} R_{T}+g_{m 1} g_{m 2} B_{1} B_{2}
$$

The pole frequency $\left(f_{0}\right)$ and quality factor $(Q)$ of the proposed filter can be found in forms:

$$
f_{0}=\frac{1}{2 \pi} \sqrt{\frac{g_{m 1} g_{m 2} B_{1} B_{2}}{C_{1} C_{2}}}, \quad Q=\frac{1}{g_{m 3} R_{T}} \sqrt{\frac{g_{m 2} C_{1}}{g_{m 1} C_{2}}}
$$

where $g_{\mathrm{m} 1}, g_{\mathrm{m} 2}, g_{\mathrm{m} 3}$ are transconductances of particular MOTA elements, $R_{\mathrm{T}}$ is the transresistance of the OTRA and $B$ is current gain of the DACA elements. From equations (2) it is obvious that $f_{0}$ of the filter can be adjusted electronically by changing values of transconductances $g_{\mathrm{m} 1}, g_{\mathrm{m} 2}$ when fulfilling a simple condition $g_{\mathrm{m} 1}=g_{\mathrm{m} 2}$ and/or by changing current gains $B_{1}, B_{2}$ when $B_{1}=B_{2}$. The quality factor of the filter can be adjusted electronically by changing values of $g_{\mathrm{m} 3}$ and/or $R_{\mathrm{T}}$. Therefore, presented topology allows two ways of the pole frequency and quality factor control. Both methods can be used independently, or they can be combined in order to extend range of control of $f_{0}$ and $Q$ (dual-parameter control).

The obtainable transfer functions of the proposed filter are described by following equations:

$$
\frac{\boldsymbol{I}_{L P+}}{\boldsymbol{I}_{I N}}=-\frac{\boldsymbol{I}_{L P-}}{\boldsymbol{I}_{I N}}=\frac{g_{m 1} g_{m 2} B_{1} B_{2}}{D(\boldsymbol{s})},
$$




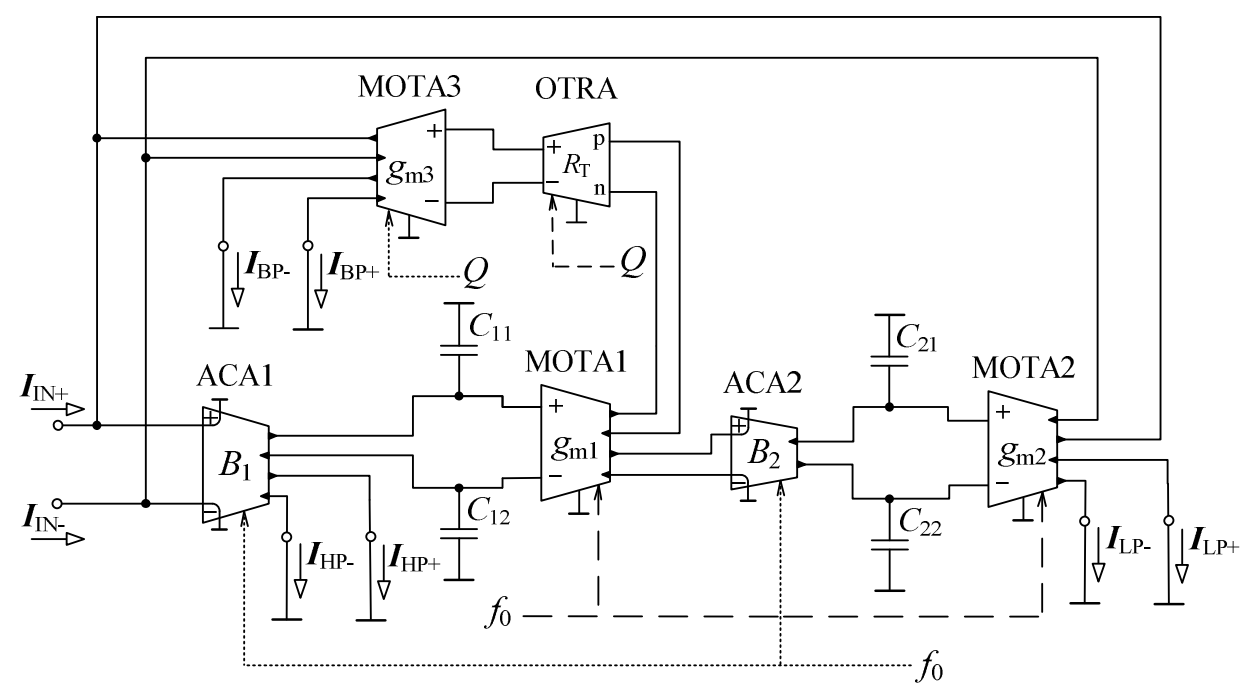

Fig. 4. Circit structure of the proposed dual-parameter control fully-differential SITO filter

$$
\begin{aligned}
& \frac{\boldsymbol{I}_{B P+}}{\boldsymbol{I}_{I N}}=-\frac{\boldsymbol{I}_{B P-}}{\boldsymbol{I}_{I N}}=\frac{\boldsymbol{s} C_{2} g_{m 1} g_{m 3} B_{1} R_{T}}{D(\boldsymbol{s})}, \\
& \frac{\boldsymbol{I}_{H P+}}{\boldsymbol{I}_{I N}}=-\frac{\boldsymbol{I}_{H P-}}{\boldsymbol{I}_{I N}}=\frac{\boldsymbol{s}^{2} C_{1} C_{2} B_{1}}{D(\boldsymbol{s})}, \\
& \frac{\boldsymbol{I}_{B R+}}{\boldsymbol{I}_{I N}}=-\frac{\boldsymbol{I}_{B R-}}{\boldsymbol{I}_{I N}}=\frac{\boldsymbol{s}^{2} C_{1} C_{2} B_{1}+g_{m 1} g_{m 2} B_{1} B_{2}}{D(\boldsymbol{s})}, \\
& \frac{\boldsymbol{I}_{A P+}}{\boldsymbol{I}_{I N}}=-\frac{\boldsymbol{I}_{A P-}}{\boldsymbol{I}_{I N}}= \\
& =\frac{\boldsymbol{s}^{2} C_{1} C_{2} B_{1}-\boldsymbol{s} C_{2} g_{m 1} g_{m 3} A_{1} R_{T}+g_{m 1} g_{m 2} B_{1} B_{2}}{D(s)}
\end{aligned}
$$

The equations (3) to (7) indicate availability of all common types of transfer functions (low-pass (LP), band-pass (BP), high-pass (HP), band-reject (BR) and all-pass (AP)). BR and AP transfer functions can be easily obtained by summing operation of particular outputs ( $\mathrm{LP}+\mathrm{HP}$ or $\mathrm{LP}+\mathrm{BP}+\mathrm{HP})$ of the circuit because the filter is of the single-input multiple-output (SIMO) type. Thus, the proposed filter is universal. All responses were taken directly from high-impedance outputs active elements.

\section{SIMULATION AND EXPERIMENTAL RESULTS}

The function of the proposed filter has been verified by PSpice simulations and experimental measurement of the implemented filter in form of a printed circuit board. Simple V/I, I/V converters and network analyzer Agilent 4395A were used for the experimental measurement. The convertors, were implemented by OPA860 [17] and OPA861 [18]. According to the way of particular implementation of individual active elements using discrete integrated circuits (UCC, UVC, EL2082) as stated in section 2, the proposed filter, in case of experimental measurement, did consist of total 7 UCC chips (supply $\pm 1, .65 \mathrm{~V}, 28 \mathrm{~mA}$ ), 2 UVC chips (same as UCC), and 2 EL2082 chip $( \pm 5 \mathrm{~V}, 15 \mathrm{~mA})$. Thus, the resulting power consumption was approximately $1.1 \mathrm{~W}$, in this particular case. We used so-called behavioral modeling using discrete chips to confirm the design correctness before rather expensive integrated circuit (IC) implementation.

Initial values of characteristic filter parameters and values of passive parts used for the simulations and experimental measurements have been selected as follows: initial values of transconductances $g_{\mathrm{m} 1}, g_{\mathrm{m} 2}$ were set to $0.5 \mathrm{mS}$, capacitors $C_{11}=C_{12}=440 \mathrm{pF}$ and $C_{21}=C_{22}=880 \mathrm{pF}$, starting values of current gains of the ACA elements were $B_{1}=B_{2}=0.25$. Thus, the calculated initial value of the pole frequency is equal to $128 \mathrm{kHz}$. The value of transconductance $g_{\mathrm{m} 3}$ has been set to $3.92 \mathrm{mS}$ and transresistance $R_{\mathrm{T}}=510 \Omega$ which results in quality factor of value 0.68 . Resistors have been used from E24 series.

Figure 5 compares simulation and experimental results of low-pass (green lines), band-pass (red lines) and high-pass (blue lines) transfer functions. The slope of attenuation of simulated high-pass transfer function is $38 \mathrm{~dB}$ per decade and the slope of attenuation of high-pass function obtained from measurement is $36 \mathrm{~dB}$ per decade. In case of BP it is 18.5 $\mathrm{dB}$ per decade for the simulation results and $17.5 \mathrm{~dB}$ per decade for the measurement results. The attenuation slope reaches $39.5 \mathrm{~dB}$ per decade for LP transfer responses in case of simulations and $37.5 \mathrm{~dB}$ per decade for the measurements. The largest difference between the simulation and experimental results can be seen at higher frequencies. The difference is given because of bandwidth limitations of used active elements and $\mathrm{V} / \mathrm{I}, \mathrm{I} / \mathrm{V}$ converters. The overpeaking at approximately $10 \mathrm{MHz}$ is caused by parasitic properties of the UCC. Most 
likely the influence of its parasitic capacitance with resistance $R_{\mathrm{T}}$ in case of the OTRA element particular implementation.

Figure 6 illustrates $f_{0}$ tunability range. Low-pass transfer function was chosen as an example. The lowest value of $f_{0}$ reaches $35.8 \mathrm{kHz}$ when $g_{\mathrm{m} 1}, g_{\mathrm{m} 2}$ were set to $0.278 \mathrm{mS}$ and $B_{1}$, $B_{2}$ to 0.1 . The transfer function does not provide unity gain in the pass-band when lower values of $f_{0}$ than indicated are set. The highest value of $f_{0}$ is $2897.1 \mathrm{kHz}$ when $g_{\mathrm{m} 1}, g_{\mathrm{m} 2}$ were set to $1 \mathrm{mS}$ and $B_{1}, B_{2}$ to 3 . The higher values of $f_{0}$, causes significant parasitic influence of tuning process on $Q$. Table II compares the theoretical values of the pole frequency with the values obtained from simulations and experimental measurements. The measured results have slightly lower values in comparison to the theoretical expectations and results of simulations. This is mainly caused by realistic characteristics of used active elements and tolerance of passive elements.

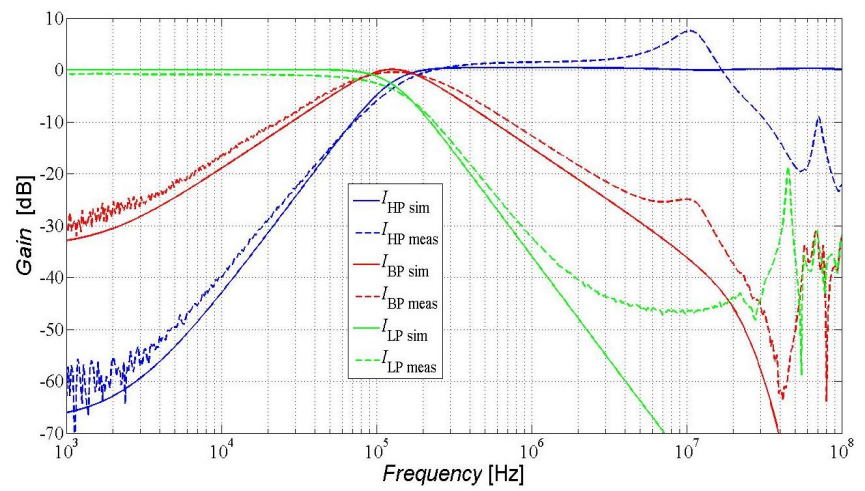

Fig. 5. Comparison of transfer functions high-pass, band-pass and low-pass of the proposed filter: simulation (solid lines) and experimental results (dashed lines)

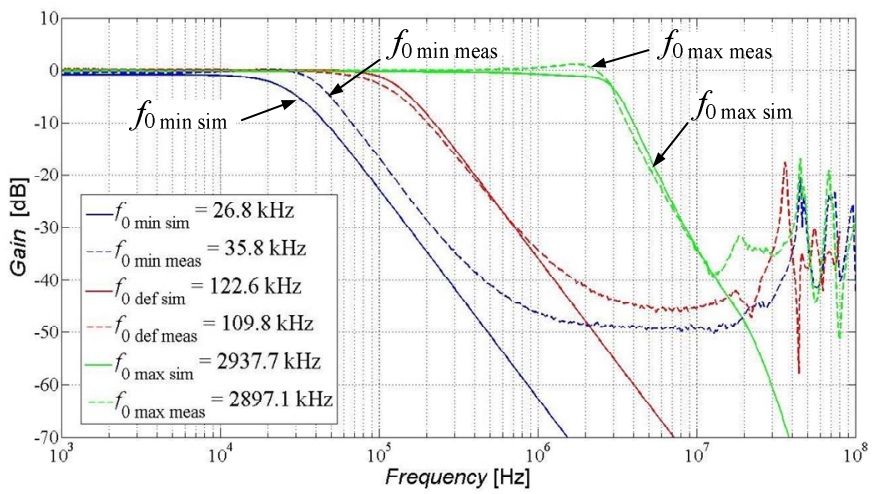

Fig. 6. Demonstration of obtainable tunability range of the pole frequency of the proposed filter in case of the LP function: simulation (solid lines) and experimental results (dashed lines)

TABLE II. COMPARISON OF THE THEORETICAL, SIMULATED AND MEASURED POLE FREQUENCIES

\begin{tabular}{|c|c|c|c|}
\hline$B[-] / g_{\mathrm{m}}[\mathrm{mS}]$ & $0.1 / 0.278$ & $0.25 / 0.5$ & $3 / 1$ \\
\hline Theoretical $f_{0}[\mathrm{kHz}]$ & 28.4 & 127.9 & 3069.3 \\
\hline Simulated $f_{0}[\mathrm{kHz}]$ & 26.8 & 122.6 & 2937.7 \\
\hline Measured $f_{0}[\mathrm{kHz}]$ & 35.8 & 109.8 & 2897.1 \\
\hline
\end{tabular}

Available tunability range of the quality factor of the proposed filter for experimental results is depicted in Fig. 7. Band-pass transfer function was selected for this test. The lowest measured $Q$ has value 0.44 when $g_{\mathrm{m} 3}$ was set to $3.92 \mathrm{mS}$ and $R_{\mathrm{T}}$ was set to $680 \Omega$. The highest obtainable $Q$ is 8.3 when $g_{\mathrm{m} 3}$ was set to $1 \mathrm{mS}$ and $R_{\mathrm{T}}$ was set to $130 \Omega$. For higher values of $Q, f_{0}$ is being shifted together with $Q$. A comparison of the theoretical, simulation and experimental values of the quality factor can be made from Table III. The values of the quality factor obtained from the experimental measurements are slightly lower than it is for theoretical values and simulation results. This is mainly caused by the reasons mentioned above.

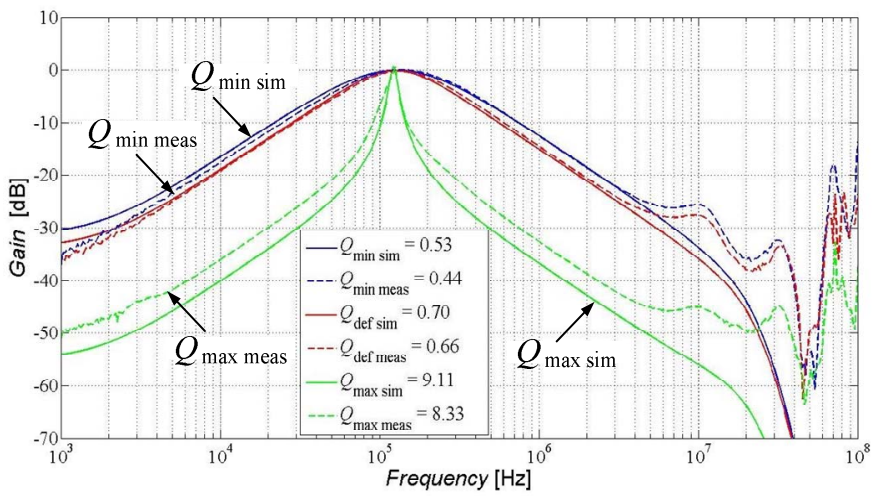

Fig. 7. Demonstration of obtainable tunability range of the quality factor of the proposed filter in case of the BP function: simulation (solid lines) and experimental results (dashed lines)

TABLE III. COMPARISON OF THE THEORETICAL, SIMULATED AND MEASURED QUALITY FACTORS

\begin{tabular}{|c|c|c|c|}
\hline$g_{\mathrm{m} 3}[\mathrm{mS}] / R_{\mathrm{T}}[\Omega]$ & $3.92 / 680$ & $3.92 / 510$ & $1 / 130$ \\
\hline Theoretical $Q[-]$ & 0.53 & 0.71 & 10.88 \\
\hline Simulated $Q[-]$ & 0.53 & 0.70 & 9.11 \\
\hline Measured $Q[-]$ & 0.44 & 0.66 & 8.33 \\
\hline
\end{tabular}

\section{CONCLUSION}

Obtained simulation and experimental results are illustrated in the paper and compared with the theoretical expectations. From the tables is evident that the simulation and experimental results are in good agreement with the theoretical results. Both simulation and experimental results prove the possibility of dual-parameter electronic control of $f_{0}$ and $Q$ of the proposed filter. The tunability range of $f_{0}$ in case of the experimental measurement was achieved between $35.8 \mathrm{kHz}$ and $2897.1 \mathrm{kHz}$ that yields $\mathrm{fmin} / \mathrm{fmax}$ ratio of 80.5 . The tunability range of $Q$ was tested from 0.44 to 8.33 which provides $Q \min / Q \max$ ratio of 18.9. Thus, the tunability range has been significantly extended in comparison to standard solutions. We also obtained good results in comparison with other filters with the dual-parameter control. 


\section{REFERENCES}

[1] J. Jerabek, J. Dvorak, R. Sotner, B. Metin, K. Vrba, "Multifunctional current-mode filter with dual-parameter control of the pole frequency," Advances in Electrical and Computer Engineering, vol. 16, issue 3, pp. 31-36, 2016.

[2] S. V. Singh, S. Maheshwari, D. S. Chauhan, "Universal currentcontrolled current-mode biquad filter employing MOCCCCTAs and grounded capacitors," Circuits and Systems, vol 1, no. 2, pp. 35-40, 2010 .

[3] A. Chaichana, A. Jantakun, M. Kumngern, W. Jaikla, "Current-mode MISO filter using CCCDTAs and grounded capacitors," Indian Journal of Pure and Applied Physics, vol. 53, no. 6, pp. 470-477, 2015.

[4] J. Jerabek, R. Sotner, N. Herencsar, J. Polak, J. Dvorak, J. Koton, "MISO Universal Frequency Filter with Dual-Parameter Control of the Pole Frequency," ELECO 2015 9th International Conference on Electrical and Electronics Engineering, 2015, pp. 24-28.

[5] L. Langhammer, J. Jerabek, J. Polak, P. Cika, "Single-Ended and FullyDifferential Current-Input Current-Output Universal Frequency Filter with Transconductace and Transresistance Amplifiers," WSEAS TRANSACTIONS on CIRCUITS and SYSTEMS, Canary Islands, Spain, 2015, pp. 56-67.

[6] J. Jerabek, R. Sotner, K. Vrba, "Tiso Adjustable Filter with Controllable Controlled-Gain Voltage Differencing Current Conveyor," Journal of Electrical Engineering, vol. 65, Issue 3, pp. 137-143, 2014.

[7] H. Chen, S. Wang, P. Li, N. Chou, C. Chang, "Single FDCCII-based current-mode universal biquadratic filter," In Proc. The 2nd International Conference Consumer Electronics, Communications and Networks (CECNet), Yichang, China, Apr. 2012, pp. 2076-2079.

[8] F. Kacar, H. Kuntman, S. Ozcan, "New High Performance CMOS Fully Differential Current Conveyor," ELECTROSCOPE Applied Electronics Number III, 2008.
[9] H. Chen, "Tunable versatile current-mode universal filter based on plustype DVCCs," AEU - International Journal of Electronics and Communications, Vol. 66, Issue 4, pp. 332-339, 2012.

[10] J. Jerabek, K. Vrba, "Comparison of the Fully-Differential and SingleEnded Solutions of the Frequency Filter with Current Followers and Adjustable Current Amplifier," In Proc. ICN2012 The Eleventh International Conference on Networks 2012, Reunion, France: IARIA, Feb. 29 - Mar. 5 2012, pp. 50-54.

[11] J. Jerabek, K. Vrba "Design of Fully Differential Filters with Basic Active Elements Working in the Current Mode," Elektrorevue, vol. 12, no.6, pp. 87-1 - 87-5, 2010.

[12] J. Jerabek, J. Koton, R. Sotner, K. Vrba, “Adjustable band-pass filter with current active elements: two fully-differential and single-ended solutions," Analog Integr. Circ. Sig. Process., vol. 74, pp. 129-139, 2013.

[13] D. Biolek, R. Senani, V. Biolkova, Z. Kolka, "Active Elements for Analog Signal Processing: Classification, Review, and New Proposals," Radioengineering, Vol. 17, No. 4, pp. 15-32, 2008.

[14] R. Sponar, K. Vrba, "Measurements and Behavioral Modelling of Modern Conveyors," International Journal of Computer Science and Network Security, vol. 3A, no. 6, pp. 57-63, 2006.

[15] N. Herencsar, J. Koton, J. Jerabek, K. Vrba, O. Cicekoglu, "VoltageMode All-Pass Filters Using Universal Voltage Conveyor and MOSFET- Based Electronic Resistors," Radioengineering, vol. 20, no. 1, pp. 10-18, 2011.

[16] EL2082 (Elantec) Current-mode multiplier (datasheet), 1996, 14 p., available online: http://www.intersil.com/content/dam/Intersil/documents/el20/el2082.pdf

[17] Texas Instruments - OPA860 - Wide Bandwidth Operational Transconductance Amplifier (datasheet). Online, http://www.ti.com/lit/ds/symlink/opa860.pdf

[18] Texas Instruments - OPA861 - Wide Bandwidth Operational Transconductance Amplifier (datasheet). Online, http://www.ti.com/lit/ds/symlink/opa861.pdf 